\title{
The clinical value of HbA1c in combination with FPG in the early screening of the elderly with type 2 diabetes
}

\author{
Lihua Liu, ${ }^{1, \#}$, Wenqing Chen ${ }^{2}$, Minghua Dong ${ }^{3, \#}$, Lixia Jiang ${ }^{4}$, Wei Qiu $^{3}$, Jian Li ${ }^{5}$, Xiaoting Luo ${ }^{1}$, \\ Zhengchun Huang ${ }^{5}$, Qin $\mathrm{Wu}^{4}$, Qinfeng $\mathrm{Wu}^{3}$, Shuiqin Chen ${ }^{1}$, Lu Ou-Yang ${ }^{3}$ and Shumei $\mathrm{Li}^{3}{ }^{3, \mathrm{a}}$ \\ ${ }^{1}$ Department of Biochemistry and Molecular Biology, Gannan Medical College, Ganzhou, Jiangxi, China \\ ${ }^{2}$ Department of Anesthesiology GanZhou Municipal Hospital, Ganzhou Jiangxi, China \\ ${ }^{3}$ Department of Epidemiology in Preventive Medicine, Gannan Medical College, Ganzhou, Jiangxi, China \\ ${ }^{4}$ Department of Clinical laboratory of 1'th affiliate hospital, Gannan Medical College, Ganzhou, Jiangxi, China \\ ${ }^{5}$ Department of Anatomy Gannan Medical College, Ganzhou, Jiangxi, China
}

\begin{abstract}
Objective: To investigate the FPG associated with HbAlc diagnosis of T2DM, to clear the best point of tangency HbAlc diagnosis of T2DM. Methods: Using simple random sampling method, from this group of T2DM field epidemiological investigation data in the database we randomly selected 300 cases of T2DM patients, 300 cases of healthy people. The sensitivity and specificity of FPG, $\mathrm{HbAlc}$ and $\mathrm{HbAlc} / \mathrm{FPG}$ combination were calculated by diagnostic screening test; Using ROC curve evaluation FPG, HbAlc and $\mathrm{HbAlc}$ in combination with FPG diagnosis value of T2DM. Results: The cutoff point of FPG for diagnosing T2DM was $6.19 \mathrm{mmol} / \mathrm{L}$, the sensitivity and specificity were respectively: $83.00 \%$, 93.67\%, area under the curve (AUC) was 0.928 ; the cutoff point of HbAlc for diagnosing T2DM was $6.40 \%$, the sensitivity and specificity were respectively: $87.00 \%, 90.33 \%$, area under the curve (AUC) is 0.935 ; Combined use of HbAlc and FPG in the diagnosis of T2DM, simultaneous detection of $\mathrm{HbAlc}$ and $\mathrm{FPG}$, when $\mathrm{HbAlc} \geq 6.40 \%$ or $\mathrm{FPG} \geq 6.19 \mathrm{mmol} / \mathrm{L}$ diagnosed with T2DM, the sensitivity and specificity were respectively: $97.79 \%, 84.61 \%$, area under the curve (AUC) is 0.960 . Conclusions: The cutoff point of $\mathrm{HbAlc}$ for diagnosing T2DM was $6.40 \%$, Combined HbA1c and FPG in the T2DM diagnosis, which can greatly improve the sensitivity, the combined application is more conducive to the early screening of T2DM.
\end{abstract}

\section{Introduction}

With the development of society by leaps and bounds, the prevalence of diabetes is in a rising trend. According to the International Diabetes Federation (IDF) latest statistics, by 2040, nearly 642 million people are expected to suffer from Diabetes, $90 \%$ to $95 \%$ of whom are in Type 2 Diabetes Mellitus(T2DM). China has now the most T2DM patients in the world [1].

Fasting Plasma Glucose (FPG) or 2 hour Postprandial Glucose(2hPG) is the most commonly used

\footnotetext{
${ }^{a}$ Corresponding author: Shumei Li, gnyxylsm@163.com. Phone: 008615083787928.

\# These authors contributed equally to this study and share first authorship.

This study was supported by scientific fund from National Natural Science Fund in China (No.81360445), Science and technology support program of Jiangxi Province (NO.20132BBG70086).
} 
method in clinical diagnosis of T2DM, But it only represents the instant moment of blood Glucose levels, and chronic hyperglycemia is the main characteristic of T2DM, So only FPG or $2 \mathrm{hPG}$ on the diagnosis of T2DM has a certain missed diagnosis. Glycated hemoglobin A1c(HbAlc) can reflect the 2-3 months of blood Glucose levels, it can be used as indicators of T2DM patients with long-term control blood Glucose. In 2010, the American Diabetes Association(ADA) took HbA1c as a new diagnostic method for T2DM[2]. However, domestic research on HbA1c as the diagnostic standard of T2DM is still in its initial stage. Because of different sample population, people's life style and the environment are different, the value of HbA1c is different. Therefore, The elderly T2DM early screening in our region and the optimal cut-off point are studied, and results will now be reported as follows.

\section{The research object and methods}

\subsection{The object of the research}

Using simple random sampling method, From this group of T2DM field epidemiological investigation database we randomly selected 300 cases of T2DM patients, 300 cases of healthy people.

\subsection{The research methods}

(1) questionnaire: Main content includes gender, age, history of smoking and drinking, and other general information; (2) laboratory tests: subjects in the morning on an empty stomach phlebotomize, subjects were unified for breakfast after blood, continue to blood after 2 hours, Biochemical parameters such as FPG, 2hPG and HbA1c were determined by Hitachi 7600 automatic biochemical analyzer.

\subsection{Statistical analysis}

Using Stata12.0 statistical software for data analysis, The sensitivity and specificity of FPG, $2 \mathrm{hPG}$ and HbA1c in the diagnosis of T2DM was calculated, using ROC curve evaluation FPG, 2hPG, HbA1c diagnosis value of T2DM.

\section{Results}

\subsection{The basic situation of the research object}

From the T2DM field epidemiological survey data of this research group, 300 cases of T2DM patients were randomly selected, and 300 healthy people were studied, average age was $60.020 \pm 9.684$ years, The maximum is 86 years old, the minimum is 38 years old, The age distribution of the study subjects was normal distribution (Table 1); The object of study the age distribution of normally distributed (Figure 1), the distribution of FPG, $2 \mathrm{hPG}$ is skewed distribution (Figure 2, Figure 3), the distribution of $\mathrm{HbA1c}$ is basic normally distributed (Figure 4).

Table 1. The basic data of subjects.

\begin{tabular}{lcccc}
\hline \multicolumn{1}{c}{ variable } & $\mathrm{n}$ & $(\bar{x} \pm s)$ & minimum & maximum \\
\hline age & 600 & $60.020 \pm 9.684$ & 38 & 86 \\
FPG & 600 & $6.813 \pm 2.359$ & 4.280 & 20.130 \\
$2 \mathrm{hPG}_{\mathrm{HbA}} \mathrm{c}$ & 600 & $11.310 \pm 5.428$ & 2.700 & 32.120 \\
\hline
\end{tabular}



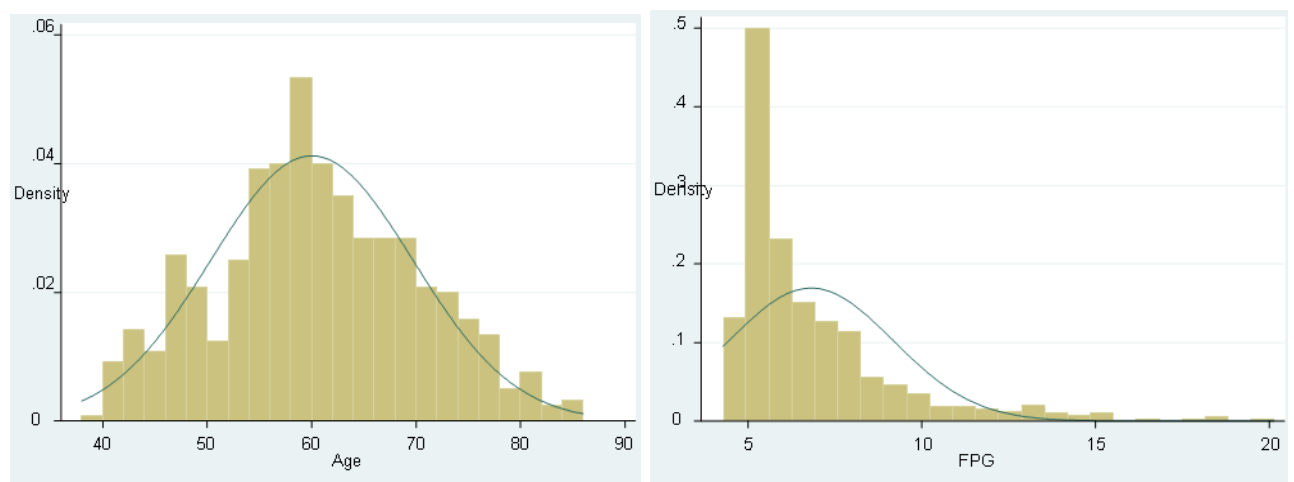

Figure 1. The age distribution of subjects.

Figure 2. Fasting plasma glucose distribution of subjects.
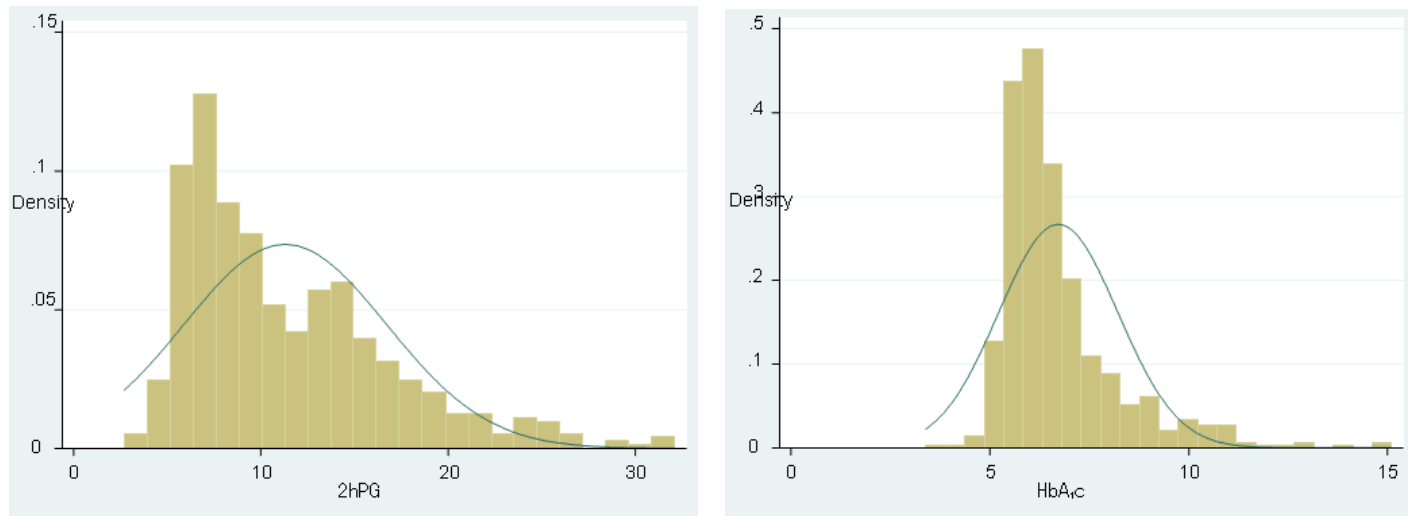

Figure 3. 2h Postprandial glucose distribution of subjects.

Figure 4. HbA1c distribution of subjects.

\subsection{To evaluate the diagnostic value of FPG, $2 \mathrm{hPG}, \mathrm{HbA1c}, \mathrm{HbA1c}$ and combined with FPG in T2DM}

\subsubsection{The sensitivity and specificity of FPG in the diagnosis of T2DM}

Application of FPG alone in the diagnosis of $\mathrm{T} 2 \mathrm{DM}$, when $\mathrm{FPG} \geq 6.19 \mathrm{mmol} / \mathrm{L}$, the sensitivity and specificity were respectively: $83 \%, 93.67 \%$ (Table 2 ).

Table 2. Sensitivity and specificity of diagnosis for T2DM using FPG

\begin{tabular}{lccccc}
\hline Cutpoint & Sensitivity & Specificity & Classified & LR+ & LR- \\
\hline$(>=4.28)$ & $100.000 \%$ & $0.000 \%$ & $50.000 \%$ & 1.000 & \\
$(>=4.39)$ & $100.000 \%$ & $0.330 \%$ & $50.170 \%$ & 1.003 & 0.000 \\
$\ldots$ & & & & & \\
$(>=6.17)$ & $83.000 \%$ & $92.670 \%$ & $87.830 \%$ & 11.318 & 0.184 \\
$(>=6.18)$ & $83.000 \%$ & $93.000 \%$ & $88.000 \%$ & 11.857 & 0.183 \\
$(>=6.19)$ & $83.000 \%$ & $93.670 \%$ & $88.330 \%$ & 13.105 & 0.182 \\
$(>=6.20)$ & $82.670 \%$ & $93.670 \%$ & $88.170 \%$ & 13.053 & 0.185 \\
$(>=6.22)$ & $82.330 \%$ & $93.670 \%$ & $88.000 \%$ & 13.000 & 0.187 \\
$\ldots$ & & & & & \\
$(>=20.13)$ & $0.330 \%$ & $100.000 \%$ & $50.170 \%$ & 0.997 & \\
$(>20.13)$ & $0.000 \%$ & $100.000 \%$ & $50.000 \%$ & 1.000 & \\
\hline
\end{tabular}




\subsubsection{The sensitivity and specificity of $2 h P G$ in the diagnosis of T2DM}

Application of $2 \mathrm{hPG}$ alone in the diagnosis of $\mathrm{T} 2 \mathrm{DM}$, when $2 \mathrm{hPG} \geq 11.18 \mathrm{mmol} / \mathrm{L}$, the sensitivity and specificity were respectively: $84.33 \%, 100.00 \%$ (Table 3 ).

Table 3. Sensitivity and specificity of diagnosis for T2DM using $2 \mathrm{hPG}$.

\begin{tabular}{|c|c|c|c|c|c|}
\hline \multirow[b]{2}{*}{ Cutpoint } & \multicolumn{5}{|c|}{ Correctly } \\
\hline & Sensitivity & Specificity & Classified & $\mathrm{LR}+$ & LR- \\
\hline$(>=2.70)$ & $100.000 \%$ & $0.000 \%$ & $50.000 \%$ & 1.000 & \\
\hline$(>=2.92)$ & $100.000 \%$ & $0.330 \%$ & $50.170 \%$ & 1.003 & 0.000 \\
\hline$(>=10.96)$ & $84.330 \%$ & $99.330 \%$ & $91.830 \%$ & 126.500 & 0.158 \\
\hline$(>=11.00)$ & $84.330 \%$ & $99.670 \%$ & $92.000 \%$ & 252.998 & 0.157 \\
\hline$(>=11.18)$ & $84.330 \%$ & $100.000 \%$ & $92.170 \%$ & 0.157 & \\
\hline$(>=11.22)$ & $84.000 \%$ & $100.000 \%$ & $92.000 \%$ & 0.160 & \\
\hline$(>=11.25)$ & $83.670 \%$ & $100.000 \%$ & $91.830 \%$ & 0.163 & \\
\hline$(>=32.12)$ & $0.330 \%$ & $100.000 \%$ & $50.170 \%$ & 0.997 & \\
\hline$(>32.12)$ & $0.000 \%$ & $100.000 \%$ & $50.000 \%$ & 1.000 & \\
\hline
\end{tabular}

\subsubsection{The sensitivity and specificity of $\mathrm{HbA} 1 \mathrm{c}$ in the diagnosis of T2DM}

Application of HbAlc alone in the diagnosis of T2DM, when HbAlc $\geq 6.40 \%$, the sensitivity and specificity were respectively: $87.00 \%, 90.33 \%$ (Table 4 ).

Table 4. Sensitivity and specificity of diagnosis for T2DM using HbAlc.

\begin{tabular}{lccccc}
\hline \multirow{2}{*}{ Cutpoint } & Sensitivity & Specificity & Classified & LR+ & LR- \\
\hline$(>=3.40)$ & $100.000 \%$ & $0.000 \%$ & $50.000 \%$ & 1.000 & \\
$(>=4.10)$ & $100.000 \%$ & $0.330 \%$ & $50.170 \%$ & 1.003 & 0.000 \\
$\ldots$ & & & & & \\
$(>=6.20)$ & $90.330 \%$ & $78.670 \%$ & $84.500 \%$ & 4.234 & 0.123 \\
$(>=6.30)$ & $88.330 \%$ & $85.670 \%$ & $87.000 \%$ & 6.163 & 0.136 \\
$(>=6.40)$ & $87.000 \%$ & $90.330 \%$ & $88.670 \%$ & 9.000 & 0.144 \\
$(>=6.50)$ & $83.000 \%$ & $93.000 \%$ & $88.000 \%$ & 11.857 & 0.183 \\
$(>=6.60)$ & $76.330 \%$ & $94.670 \%$ & $85.500 \%$ & 14.313 & 0.250 \\
$\ldots$ & & & & & \\
$(>=15.1)$ & $0.33 \%$ & $100.00 \%$ & $50.17 \%$ & 0.9967 & \\
$(>15.1)$ & $0.00 \%$ & $100.00 \%$ & $50.00 \%$ & 1.0000 & \\
\hline
\end{tabular}

\subsubsection{The sensitivity and specificity of HbA1c combined with FPG in the diagnosis of T2DM}

When $\mathrm{HbAlc} \geq 6.40 \%, \mathrm{HbA} 1 \mathrm{c}$ screening test is positive, or $\mathrm{FPG} \geq 6.19 \mathrm{mmol} / \mathrm{L}$ for screening test is positive, combined use of $\mathrm{HbA1c}$ and FPG, namely $\mathrm{HbA1c} \geq 6.40 \%$ or $\mathrm{FPG} \geq 6.19 \mathrm{mmol} / \mathrm{L}$ in the two indicators as long as there is a positive, and then combined screening test is positive. from the table(24), combined use of $\mathrm{HbA} 1 \mathrm{c}$ and FPG diagnosis of T2DM, when $\mathrm{HbA} 1 \mathrm{c} \geq 6.40 \%$ or $\mathrm{FPG} \geq 6.19 \mathrm{mmol} / \mathrm{L}$, which can calculate the sensitivity and specificity were respectively: 97.79\%, 84.61\%.

\subsection{FPG, 2hPG, HbA1c, combination HbA1c and FPG ROC curve}

From application of FPG, 2hPG, HbAlc diagnostic screening test, you can see that separate 
application of FPG, 2hPG, HbA1c, the area under the curve AUC were respectively : 0.928, 0.959, $0.935,95 \%$ ci respectively: $(0.905,0.950),(0.943,0.975),(0.915,0.955)$; HbA1c combined with FPG area under the curve AUC was $0.960,95 \%$ CI $(0.944,0.976)$; Single application of FPG, $2 \mathrm{hPG}$, $\mathrm{HbAlc}$, and HbAlc combined with FPG diagnostic screening test area under the curve orderly was HbAlc+FPG $>2 \mathrm{hPG}>\mathrm{HbA1c}>$ FPG $(0.960>0.959>0.935>0.928)$, They have statistical significance ( Table 5, Figure 5).

Table 5. ROC area in FPG, 2hPG, HbA1c and combination HbA1c and FPG in diagnosis of T2DM.

\begin{tabular}{ccccc}
\hline & & ROC & -Asymptotic Normal- \\
variable name & Obs & $\operatorname{Area}(\bar{x} \pm s)$ & 0.905 & 0.950 \\
FPG & 600 & $0.928 \pm 0.012$ & 0.943 & 0.975 \\
2hPG & 600 & $0.959 \pm 0.008$ & 0.915 & 0.955 \\
HbA1c & 600 & $0.935 \pm 0.010$ & 0.944 & 0.976 \\
HbA1c+FPG & 600 & $0.960 \pm 0.008$ & & \\
\hline
\end{tabular}

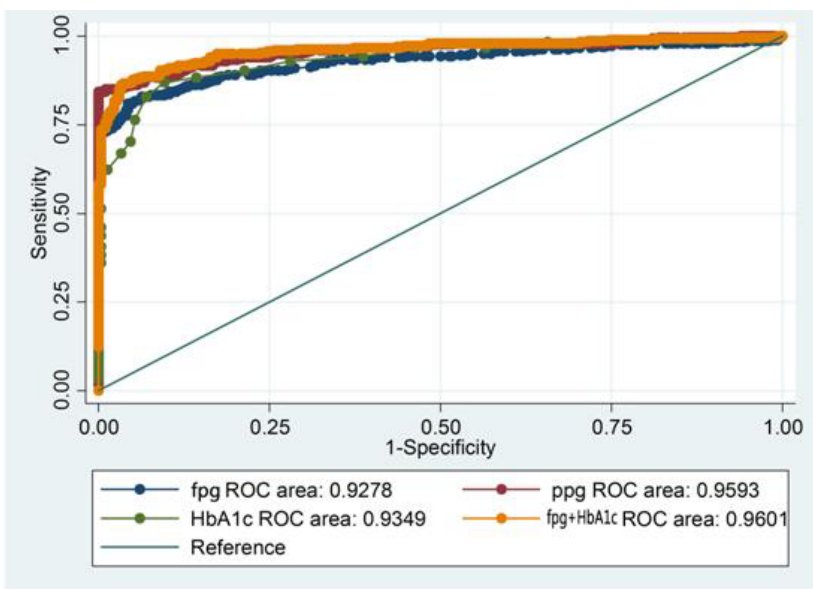

Figure 5. ROC curve of FPG, 2hPG, HbAlc and combination HbA1c and FPG in diagnosis of T2DM.

\section{Discussion}

\subsection{HbA1c the clinical diagnostic value and the best point of contact}

Though T2DM in China has increased dramatically in recent years, yet a lot of T2DM patients were not diagnosed early. HbA1c as T2DM in Europe and the United States has been one of the standards of diagnosis. Selvin [3] research shows that the coefficient of variation of $2 \mathrm{hPG}$, FPG and HbA1C shows a decreasing trend $(16.7 \%>5.7 \%>1.7 \%)$, The advantages of HbAlc coefficient of variation are small, so HbA1c is more reproducible than FPG as a tool for the diagnosis of T2DM. HbA1c can be done at any time, and do not require patients to fast or do any preparation. Furthermore FPG, $2 \mathrm{hPG}$ lifestyle changes would be a short time, and HbAlc status is not affected by diet, movement and whether the effects of taking certain medications, so it can be a reliable basis for monitoring of blood glucose control is good or not. So ADA in 2010 and in diabetes guidelines issued the $\mathrm{HbA} 1 \mathrm{c} \geq 6.5 \%$ as one of the diagnostic criteria for T2DM.

Although HbAlc in the diagnosis of T2DM has more advantages than the FPG, $2 \mathrm{hPG}$, there are ethnic and regional differences, which followed by a lot of T2DM researchers according to different ethnic regions, HbA1c application study on a large number of experiments in early diagnosis of T2DM, HbA1c and ADA in the diagnosis of the T2DM patients the best cut-off points were compared. 
In the multi-ethnic population of Canada, the best cut-off value of HbAlc was $5.9 \%$, the sensitivity and specificity were 75\% and 79.1\%[4]. In South Korea in 2012 a study also showed that people in the area of diagnosed T2DM HbA1c and the best cut-off value was 5.95\%. The sensitivity of 77.0\% and a specificity of $89.4 \%$ [5]. In some areas of our country, HbAlc has been used in the early diagnosis of T2DM in community population. BaoY et al[6] Study on the community population in Shanghai showed that when the HbA1c was $6.3 \%$, the sensitivity and specificity were $62.8 \%$ and 96.1\% respectively. Zhou XH[7] on the Qingdao community population study found that when $\mathrm{HbA} 1 \mathrm{c}$ was $6 \%$, the sensitivity and specificity of screening T2DM reached the best balance point. Ye Xiangjun et al [8] research shows that using HbAlc in patients with T2DM diagnosed the best cut-off value was $6.35 \%$. The sensitivity and specificity were $80 \%$ and $89 \%$.

In this study, 300 male and female subjects were studied, the number of subjects who are 40 to 64 years accounted for $68.45 \%$, the number of subjects who are 65 years old and above accounted for $31.55 \%$. the cutoff point of FPG for diagnosing T2DM was $6.19 \mathrm{mmol} / \mathrm{L}$, with sensitivity and specific degrees respectively: $83.00 \%$ and $93.67 \%$; the cutoff point of $2 \mathrm{hPG}$ for diagnosing T2DM was 11.18 $\mathrm{mmol} / \mathrm{L}$, with sensitivity and specific degrees respectively: $84.33 \%$ and $100.00 \%$; the cutoff point of HbA1c for diagnosing T2DM was $6.40 \%$, with sensitivity and specific degrees respectively: $87.00 \%$ and $90.33 \%$; The corresponding rate of missed diagnosis was $17 \%, 15.67 \%$ and $13 \%$, which further showed that the rate of missed diagnosis of HbAlc was lower than that of FPG and $2 \mathrm{hPG}$ in the diagnosis of T2DM. The Relationship of ROC area for $\mathrm{AUC}_{2 \mathrm{hPG}}>\mathrm{AUC}_{\mathrm{HbAlc}}>\mathrm{AUC}_{\mathrm{FPG}}(0.935>$ $0.959>0.959$ ), Tips for early diagnosis of T2DM, HbA1c than application FPG has higher diagnosis rate, Although application $2 \mathrm{hPG}$ to diagnosis sensitivity and specificity of T2DM more high, $2 \mathrm{hPG}$ has operation problem, poor repeatability and long time, detection result is easy to change, considering application HbA1c as T2DM index of early diagnosis is more effective.

In this study, when $\mathrm{HbA} 1 \mathrm{c}$ is $6.4 \%$, its sensitivity and specificity were the best, which was close to the research results of BaoY[6] and so on, which may be due to we are in the old revolutionary base areas, compared to other developed cities, economic development is relatively backward here, the majority of samples are older workers and farmers, and they like salty, spicy as well as pickled products and their health consciousness is not strong. Some may early suffer from T2DM, because they lack regular physical examination, they did not find T2DM in time to prevent intervention as soon as possible.

\subsection{Value of combined application of HbA1c and FPG in the diagnosis of T2DM}

$2 \mathrm{hPG}$ is currently the "gold" standard in the diagnosis of T2DM, but it consumes long time and more energy, and it is tedious in operation and poor in repeatability, so it does not apply to large-scale epidemiological survey. Separately using HbA1c to early diagnosis of T2DM may also cause misdiagnosis, but if combine HbA1C with FPG for early diagnosis of T2DM will greatly improve the sensitivity of diagnosis and reduce the rate of misdiagnosis. At present, many abroad studies Has also suggested that a combination of HbA1c and FPG for early diagnosis of T2DM. Such as Yoshihik [9] according to a study in the samples of 109 patients diagnosed with T2DM combined HbA1c and FPG test, finding that 18 patients with T2DM FPG is normal, and HbAlc is higher than normal, so a separate use FPG misdiagnosis in early diagnosis of T2DM has certain, Combination use of HbA1c and FPG in early diagnosis of T2DM, which can reduce the rate of missed diagnosis of T2DM. besides, the results of Kim KS et al[10] show that the sensitivity can reach $95.5 \%$ when combine $\mathrm{HbA} 1 \mathrm{c} \geq 6.1 \%$ and $\mathrm{FPG} \geq 6.1 \mathrm{mmol} / \mathrm{L}$ for early diagnosis of $\mathrm{T} 2 \mathrm{DM}$, which is much higher than any single application of $\mathrm{HbA} 1 \mathrm{c} \geq 6.1 \%$ or separate application of $\mathrm{FPG} \geq 6.1 \mathrm{mmol} / \mathrm{L}$, thus the rate of misdiagnosis will be reduced misdiagnosis. China survey found that heart disease of coronary heart disease an important with disease or diabetes, mostly T2DM, but for many patients with coronary heart disease FPG is normal or near normal, but the rise in blood glucose after load, that is to say after the OGTT experiment $2 \mathrm{hPG}$ blood glucose is very high, and the patients with coronary heart disease only by FPG to diagnose T2DM or pre diabetes would result 75 percent of the rate of missed diagnosis, for the people of the disease have to be tested by combination with HbA1c and FPG [11]. 
$\mathrm{Hu} \mathrm{Y}$ [12] to high-risk groups such as screening for T2DM research shows that 2298 from ruijin hospital check-up crowd as the research object, when the HbA1c was $6.1 \%$ and a separate FPG was $6.1 \mathrm{mmol} / \mathrm{L}$, the sensitivity and speciality was also 81\%; But if HbAlc and FPG combined application in the early diagnosis of T2DM, which greatly improved the sensitivity and specificity. When HbA1c was $6.1 \%$ and FPG is $6.1 \mathrm{mmol} / \mathrm{L}$, the sensitivity and specificity of early diagnosis of T2DM were increased to $96.5 \%, 96.3 \%$ respectively, Their combination can also be used to screen for impaired glucose, with a sensitivity of $82.4 \%$. There are a lot of research is also supported by the combination of HbA1c and FPG to diagnose T2DM [13, 14].

Our study separately analyzes the sensitivity of FPG and HbA1c when diagnosing T2DM , respectively $83.00 \%, 87.00 \%$, It is suggested that both FPG and HbA1c may cause missed diagnosis and the missed diagnosis rate is $17 \%$ and $13 \%$ respectively; Combined use of $\mathrm{HbA} 1 \mathrm{c}$ and FPG diagnosis of T2DM, simultaneous detection of HbA1c and FPG, when HbA1c $\geq 6.40 \%$ or FPG $\geq 6.19$ $\mathrm{mmol} / \mathrm{L}$ diagnosed with T2DM, the sensitivity was $97.79 \%$, the missed diagnosis of just $2.21 \%$. So united $\mathrm{HbA} 1 \mathrm{c}$ and FPG can improve the sensitivity of T2DM in early diagnosis, reduce missed diagnosis. In this study area under the ROC curve is greater than 0.9 , and the area of the largest is the combined use of HbA1c and FPG, so the combined use of HbA1c and FPG diagnosed T2DM diagnosis rate is highest.

The combined application of HbA1c and FPG has better sensitivity and specificity in the diagnosis of T2DM, On early detection, early diagnosis and early treatment of T2DM combined use of HbA1c and FPG has good effect, and can once pump blood and get two a different of results, in other words, it can early diagnosis whether with T2DM, which reduces patients' and medical personnels' trouble of two times pumping blood, which also avoids separately application FPG in the diagnosis of T2DM which needed more trouble of repeatly pumping blood. Combined application can reflect the situation of glucose metabolism from two different levels of the instant instant blood glucose level and the relative long-term blood glucose level, and it is a convenient and effective method for early diagnosis of $\mathrm{T} 2 \mathrm{DM}$ in the elder.

\section{References}

1. Yang WY, Lu JM, Weng JP, Jia WP, Ji LN, Xiao JZ, N Englo J Med, Prevalence of diabetes among men and women in China, 362,1090-1101(2010).

2. Saudek CD, Herman WH, Sacks DB, Bergenstal RM, Edelman D, Clin Endoerinol Metab, A new look at screening and diagnosing diabetes mellitus, 93,2447-2453(2008).

3. Selvin E, Crainiceanu CM, Brancati FL, Coresh J, Arch Intern Med, Short-term variability in measures of glycemia and implications for the classification of diabetes, 167,1545-1551(2007).

4. Herman WH, Ma Y, Uwaifo G, Haffner S, Kahn SE, Horton ES, Diabetes Care, Differences in $A 1 C$ by race and ethnicity among patients with impaired glucose tolerance in the Diabetes Prevention Program, 30,2753-2757(2007).

5. Kim JH, Kim GW, Lee MY, Shin YG, Koh SB, Diabetes Metab J, Role of $H b A_{l} c$ in the screening of Diabetes Mellitus in a Korean Rural Community, 36,37-42(2012).

6. BaoY, Ma X, Li H, Zhou M, Hu C, Wu HY, BMJ, Glycated hemoglobin $A_{1} c$ for diagnosing diabetes in Chinese population: cross sectional epidemiological survey, 340,c2249(2010).

7. Zhou X, Pang Z, Gao W, Diabetes Care, Performance of an A1C and fasting capillary blood glucose test for screening newly diagnosed diabetes and pre-diabetes defined by an oral glucose tolerance test in Qingdao, China, 33,545-550(2010).

8. Ye XQ, Lan XC, Xu GL, Prevention and Treatment of Cardio-Cerebral-vascular Disease, The value of HbAlc in the diagnosis of T2DM, 12,306-308(2012).

9. Yoshihik U, Steffes MW, Clin Chem, A primary predictor of the development or reversal of complications of diabetes mellitus, 47,1157(2001).

10. Kim KS, Kim SK, Lee YK, Diabetic Medicine, Diagnostic value of glycated haemoglobin HbA $A_{I} c$ for the early detection of diabetes in high--risk subjects, 25,997-1000(2008).

11. Diabetes branch of Chinese Medical Association, Chinese Journal of Diabetes Mellitus, Guidelines 
for prevention and treatment of type 2 diabetes in China (2013 Edition), 6,447-498(2014).

12. Hu Y, Liu W, Chen Y, Zhang M, Wang LH, Zhou H, Wu PH, Acta Diabetol, Combined use of fasting plasma glucose and glycated hemoglobin Alc in the screening of diabetes and impaired glucose tolerance, 47,231-236(2010).

13. Herman WH, Fajans SS, Pol Arch Med Wewn, Hemoglobin Alc for the diagnosis of diabetes: practical considerations, 120,37-40(2010).

14. Inoue K, Matsumoto M, Kobayashi Y, Diabetes Res Clin Pract, The combination of fasting plasma glucose and glycosylated hemoglobin predicts type 2 diabetes in Japanese workers, 77, 451-458(2007). 